\title{
Effect of microscopic damage events on static and ballistic impact strength of triaxial braid composites
}

\author{
Justin D. Littell, Wieslaw K. Binienda, and William A. Arnold \\ The University of Akron, Akron, $\mathrm{OH}$ \\ Gary D. Roberts and Robert K. Goldberg \\ NASA Glenn Research Center, Cleveland, $\mathrm{OH}$
}

In previous work, the ballistic impact resistance of triaxial braided carbon/epoxy composites made with large flat tows (12k and $24 k$ ) was examined by impacting 2'X2'X0.125" composite panels with gelatin projectiles. Several high strength, intermediate modulus carbon fibers were used in combination with both untoughened and toughened matrix materials. A wide range of penetration thresholds were measured for the various fiber/matrix combinations. However, there was no clear relationship between the penetration threshold and the properties of the constituents. During some of these experiments high speed cameras were used to view the failure process, and full-field strain measurements were made to determine the strain at the onset of failure. However, these experiments provided only limited insight into the microscopic failure processes responsible for the wide range of impact resistance observed.

In order to investigate potential microscopic failure processes in more detail, quasi-static tests were performed in tension, compression, and shear. Full-field strain measurement techniques were used to identify local regions of high strain resulting from microscopic failures. Microscopic failure events near the specimen surface, such as splitting of fiber bundles in surface plies, were easily identified. Subsurface damage, such as fiber fracture or fiber bundle splitting, could be identified by its effect on in-plane surface strains. Subsurface delamination could be detected as an out-of-plane deflection at the surface. Using this data, failure criteria could be established at the fiber tow level for use in analysis. An analytical formulation was developed to allow the microscopic failure criteria to be used in place of macroscopic properties as input to simulations performed using the commercial explicit finite element code, LS-DYNA. The test methods developed to investigate microscopic failure will be presented along with methods for determining local failure criteria that can be used in analysis. Results of simulations performed using LS-DYNA will be presented to illustrate the capabilities and limitations for simulating failure during quasi-static deformation and during ballistic impact of large unit cell size triaxial braid composites. 
Effect of Microscopic Damage Events on Static and Ballistic Impact Strength of Triaxial Braid Composites

CompTest Conference - Oct. 21, 2008

Dayton, Ohio

\author{
Justin Littell \\ Ph.D. Student \\ University of Akron \\ Akron, Ohio
}

Wieslaw K. Binienda, William A. Arnold - University of Akron, Akron Ohio Gary D. Roberts, Robert K. Goldberg - NASA Glenn Research Center, Cleveland Ohio 


\section{Triaxial Braided Composite Materials}

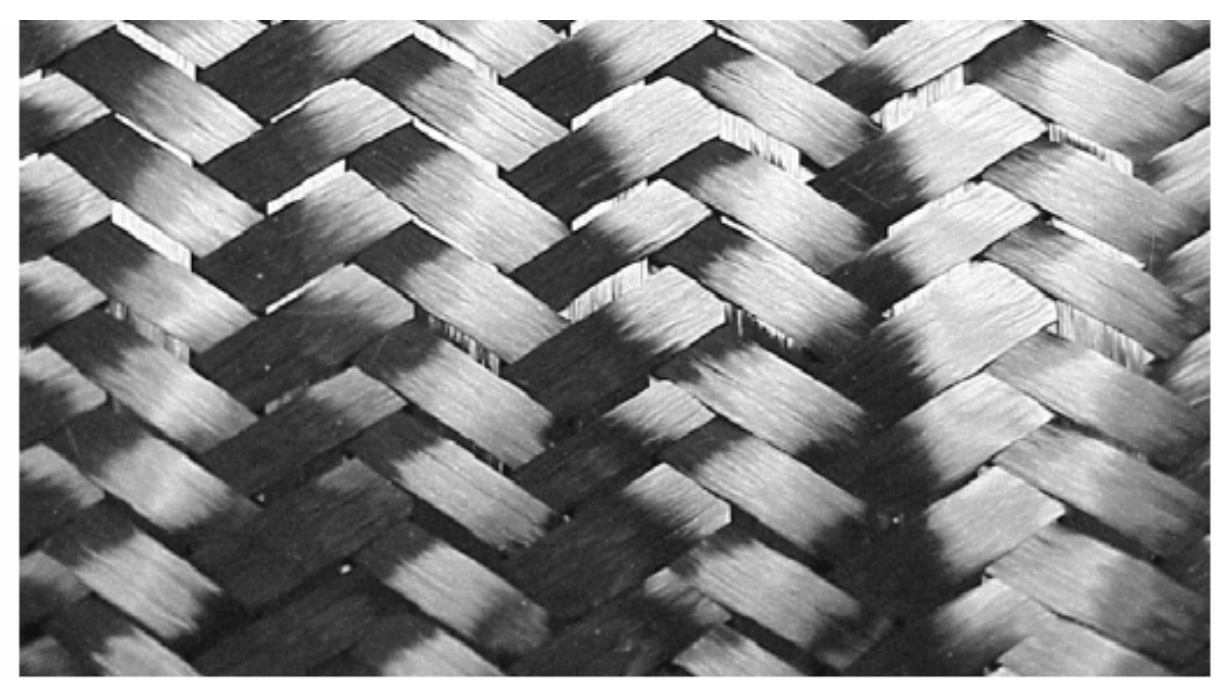

Axial Fiber Direction

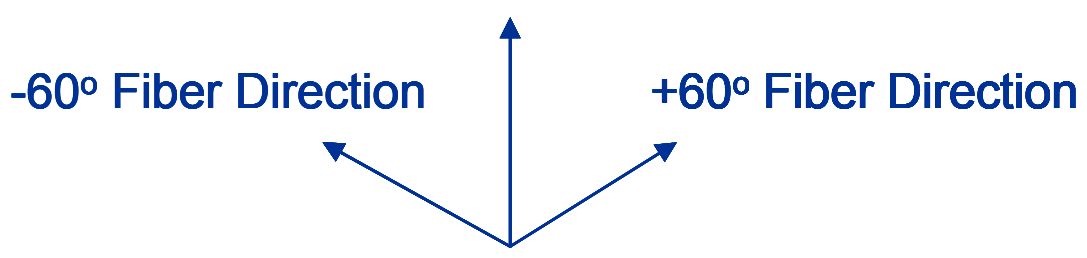

- Two dimensional triaxial braid

- $24 \mathrm{k}$ wide axial and $12 \mathrm{k}$ wide bias fiber tows

- Layers of $+60^{\circ}$ and $-60^{\circ}$ bias fibers braided over a $0^{\circ}$ axial fiber

- Quasi-isotropic architecture

- Layup of 6 Layers of braid, total composite thickness 0.125 "

- Resin Transfer Molding process (RTM)

- Volume fraction of $56 \%$ nominal 


\section{Materials}

- High Strength, Standard Modulus Fiber - Toray T700

- Two resins

- Toughened Cytec Cycom ® PR520

- Untoughened Hexcel 3502
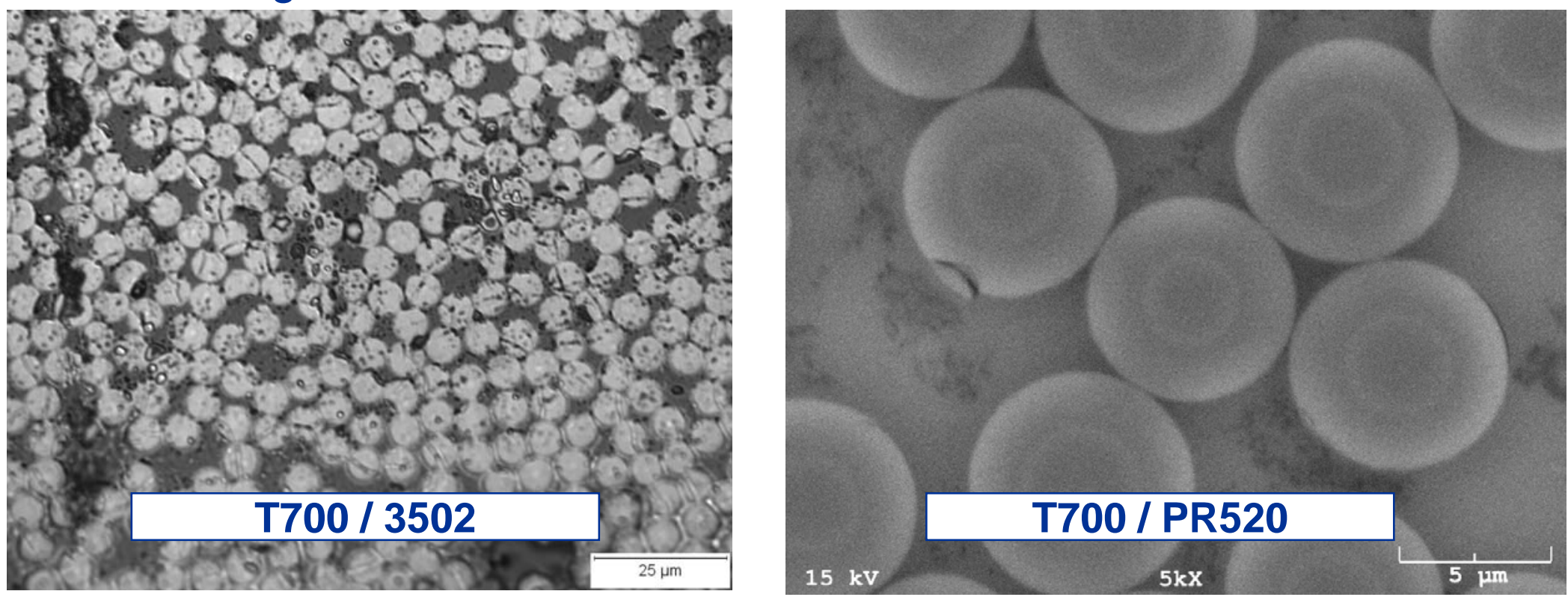

- Presented as examples to cover range of material response 


\section{Model Development}

- Testing and modeling were done in parallel

- Test data used optical measurement techniques to obtain full field strain data

- Quasi-static Testing

- By examining the test data and using classical composite theory, a new approach was developed to model composites using a novel "Subcell" approach

- Modeling data needs

- Composite section properties - braid geometry

- Composite material properties - test data

- Models developed in LS-DYNA

- Transient, nonlinear, explicit finite element code

- Primarily impact loading that composites will be subjected 


\section{Photogrammetry used for Data Collection}
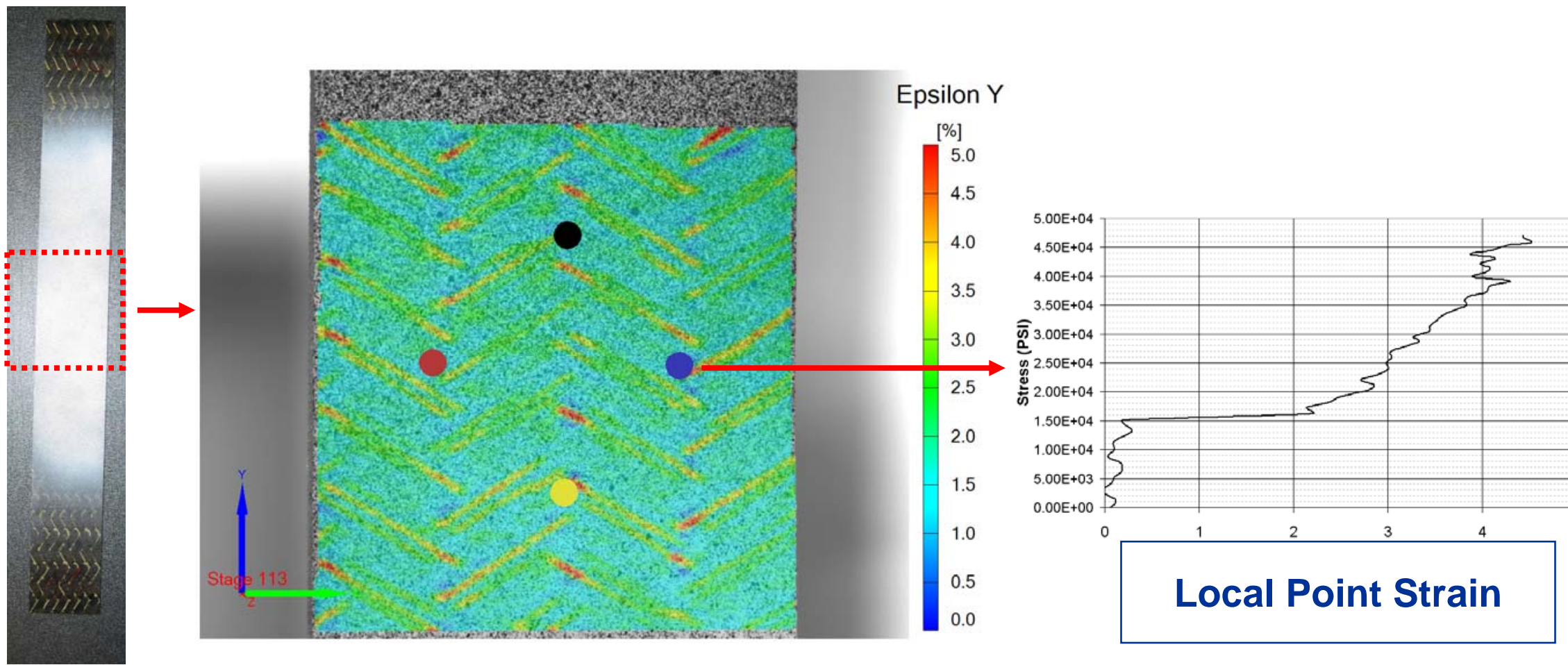

- Global stress vs. strain curves found by creating a "digital strain gage"

- Measures material response in specific areas on specimen

- Seen by noting lines of high localized strain

- Local failure mechanisms and deformations must be accounted for when developing an analytical method 


\section{Triaxial Braided Model Methodology}

- Develop a macromechanical finite element model capable of capturing the braid architecture and material properties of triaxially braided composites

- Layers of unidirectional lamina stacked in a "Subcell" configuration

- Needs local lamina level modulus and failure properties

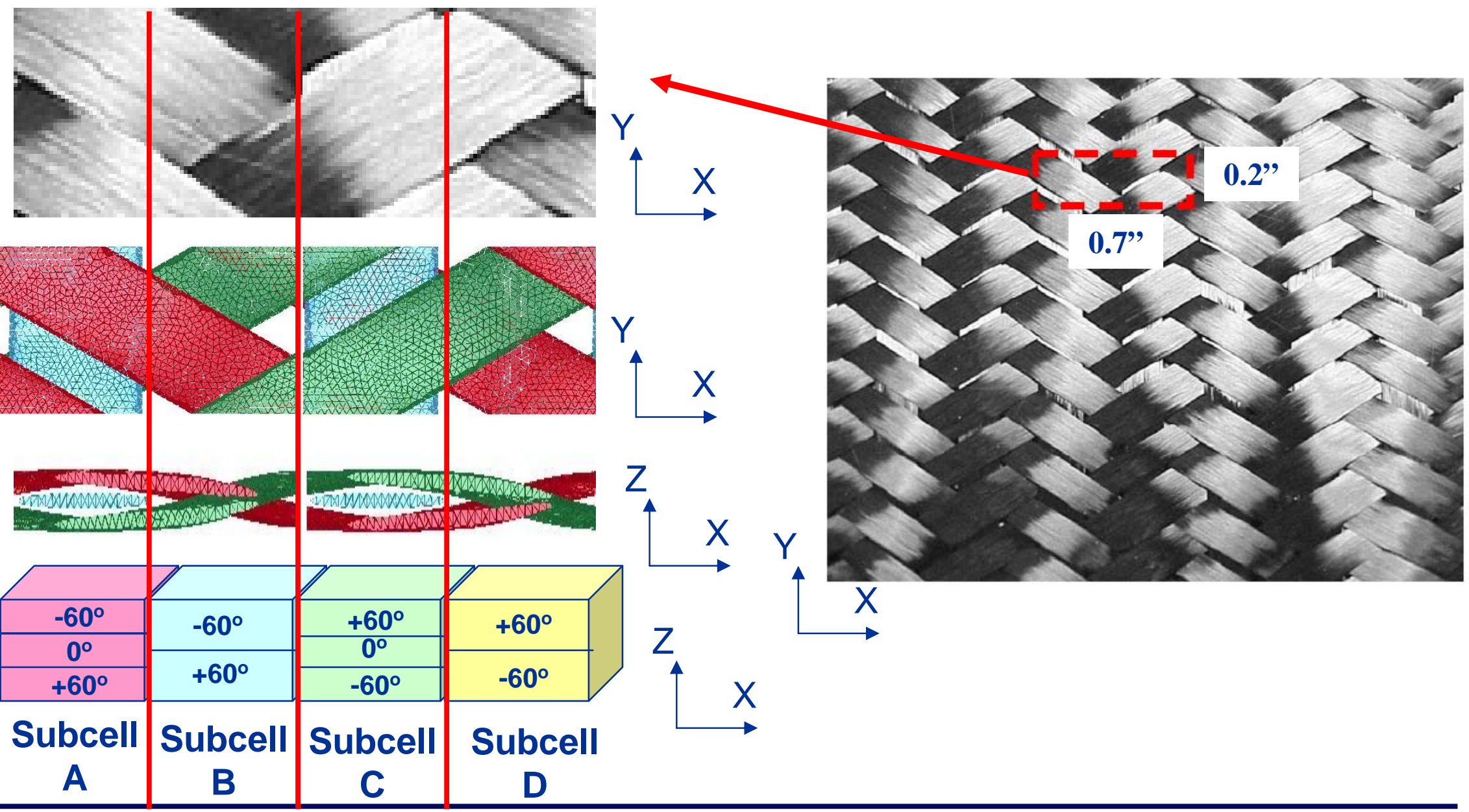




\section{Defining the Braid Geometry (Section)}

- Each part has unique section properties

- Each section contains information about number of layers (15) and braid angle $(\Theta)$ at each layer

- Braid was modeled as layers of unidirectional lamina

- Shifted (Idealized)

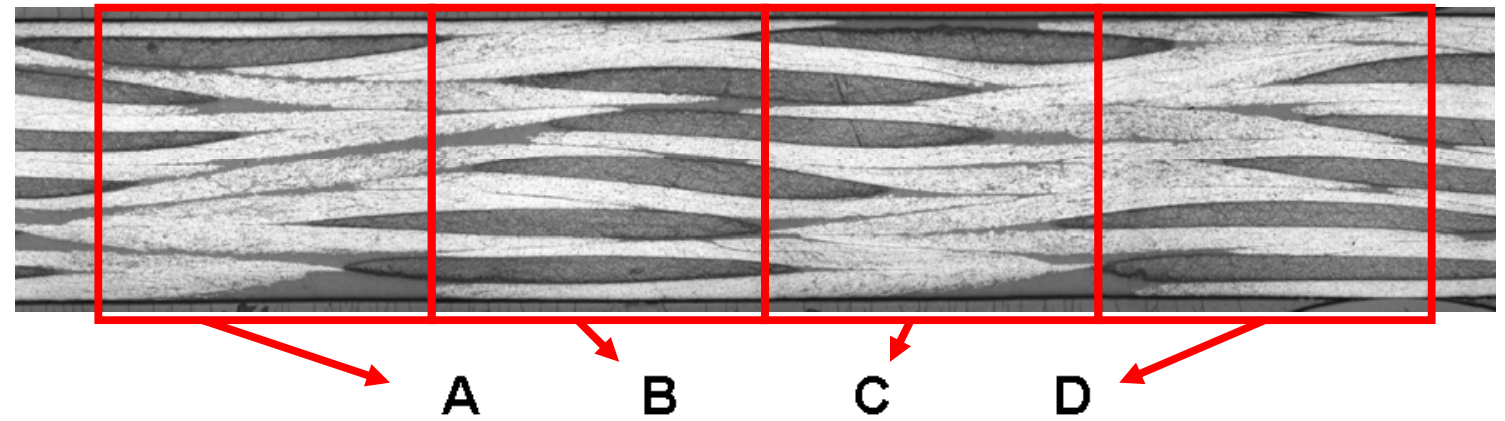

\begin{tabular}{|c|c|c|c|}
\hline$-60^{\circ}$ & \multirow{2}{*}{$+60^{\circ}$} & $+60^{\circ}$ & \multirow{2}{*}{$+60^{\circ}$} \\
\hline $0^{\circ}$ & & $0^{\circ}$ & \\
\hline$+60^{\circ}$ & $-60^{\circ}$ & $-60^{\circ}$ & $-60^{\circ}$ \\
\hline \multirow{2}{*}{$+60^{\circ}$} & $+60^{\circ}$ & \multirow{2}{*}{$+60^{\circ}$} & $-60^{\circ}$ \\
\hline & $0^{\circ}$ & & $0^{\circ}$ \\
\hline$-60^{\circ}$ & $-60^{\circ}$ & $-60^{\circ}$ & $+60^{\circ}$ \\
\hline$+60^{\circ}$ & \multirow[t]{2}{*}{$+60^{\circ}$} & $-60^{\circ}$ & \multirow[t]{2}{*}{$+60^{\circ}$} \\
\hline $0^{\circ}$ & & $0^{\circ}$ & \\
\hline$-60^{\circ}$ & $-60^{\circ}$ & $+60^{\circ}$ & $-60^{\circ}$ \\
\hline \multirow[t]{2}{*}{$+60^{\circ}$} & $-60^{\circ}$ & \multirow[t]{2}{*}{$+60^{\circ}$} & $+60^{\circ}$ \\
\hline & $0^{\circ}$ & & $0^{\circ}$ \\
\hline$-60^{\circ}$ & $+60^{\circ}$ & $-60^{\circ}$ & $-60^{\circ}$ \\
\hline
\end{tabular}




\section{A New Methodology Developed for Implementation of Material Properties}

Obtain Fiber, Matrix Properties<smiles>C1CCCC1</smiles>

Micromechanics

- Develop effective lamina properties<smiles>C1CCCC1</smiles>

\section{CLPT}

- Develop effective laminate properties<smiles>C1CCCC1</smiles>

\section{Develop a FEM}

(One layer, homogenized)

Traditional Approach (Bottom - Up)

- Items needed

- Fiber properties - Assumed

- Matrix properties

- Stackup sequence
Obtain Composite stresses, strains

From optical measurements

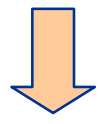

\section{CLPT}

- Back out effective laminate properties

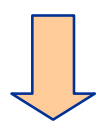

Micromechanics

- Back out effective lamina properties

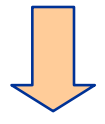

Develop a FEM

(includes layers and subcell props)

New Approach

(Top - Down)

- Items needed

- COMPOSITE TEST DATA

- Stackup sequence 


\section{Material Card \\ MAT_RATE_SENSITIVE_COMPOSITE_FABRIC}

\$\$\$ USING AS TESTED T700s/PR520 Material properties and mat 158 *MAT_RATE_SENSITIVE_COMPOSTTE_EARRTR

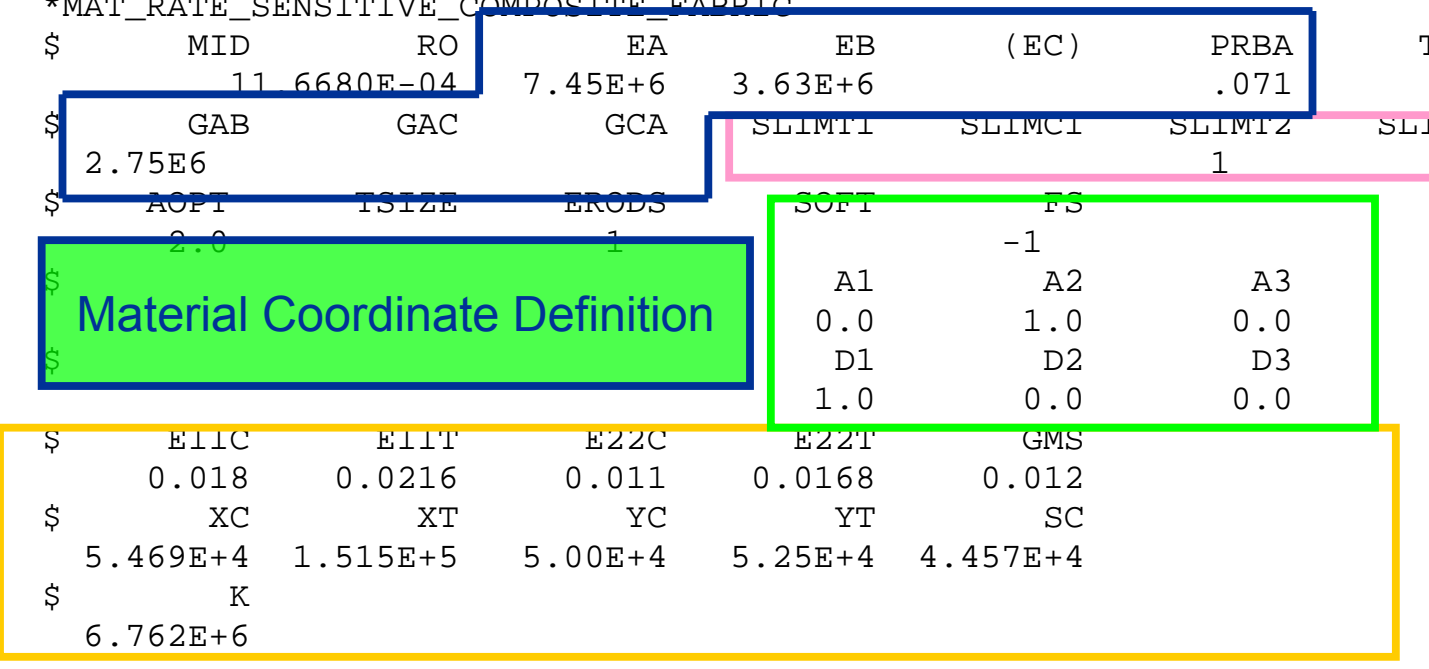

TAU1
GAMMA1

Controls elastic/plastic behavior of failure

\begin{tabular}{|l|l|}
\hline \multicolumn{2}{|l|}{ Material Response Properties } \\
\hline Property & Value \\
\hline EA & Axial Modulus \\
\hline EB & Trans. Modulus \\
\hline PRBA & In Plane Poisson \\
\hline GAB & In Plane Shear Mod. \\
\hline
\end{tabular}

\begin{tabular}{|l|l|l|l|}
\hline \multicolumn{3}{|c|}{ Failure Properties } \\
\hline Property & Name & Property & Value \\
\hline E11C & Comp. strain (Ax.) & XC & Comp. strength (Ax.) \\
\hline E11T & Tens. Strain (Ax.) & XT & Tens. Strength (Ax.) \\
\hline E22C & Comp. strain (Trans.) & YC & Comp. Strength (Trans.) \\
\hline E22T & Tens. strain (Trans.) & YT & Tens. Strength (Trans.) \\
\hline GMS & Shear strain & SC & Shear Strength \\
\hline
\end{tabular}




\section{Classical Laminated Plate Theory (CLPT) Use in Reverse for Top-Down Approach}

- Stresses/Surface Tractions are related to strains by the following

$$
\left[\begin{array}{l}
{[N]} \\
{[M]}
\end{array}\right]=\left[\begin{array}{ll}
{[A]} & {[B]} \\
{[B]} & {[D]}
\end{array}\right]\left[\begin{array}{l}
{[\varepsilon]} \\
{[\gamma]}
\end{array}\right]
$$

- Balanced and Symmetric (B and D matrices, also A16, A26 = 0)

$\left[\begin{array}{l}N x \\ N y \\ 0\end{array}\right]=\left[\begin{array}{rrr}A 11 & A 12 & 0 \\ A 12 & A 22 & 0 \\ 0 & 0 & A 66\end{array}\right]\left[\begin{array}{l}\varepsilon_{x} \\ \varepsilon_{y} \\ 0\end{array}\right]$

- Where

$$
\begin{aligned}
& A 11=\sum \bar{Q} 11=\bar{Q} 11^{0 \mathrm{deg}} * t^{\text {ddeg }}+\bar{Q} 11^{60 \mathrm{deg}} * t^{60 \mathrm{deg}}+\bar{Q} 11^{-60 \mathrm{deg}} * t^{-60 \mathrm{deg}} \\
& \bar{Q} 11=m^{4} Q 11+n^{4} Q 22+2 m^{2} n^{2} Q 12+4 m^{2} n^{2} Q 66
\end{aligned}
$$$$
Q 11=\frac{E 11}{1-v 12 * v 21}, Q 22=\frac{E 22}{1-v 12 * v 21}, Q 12=\frac{v 21 * E 11}{1-v 12 * v 21}, Q 66=G 12
$$

- E11, E22, v12, v21, G12 are parameters needed in LS-DYNA 


\section{Equation Development}

\section{Micromechanics of Composite Materials assumptions}

- Transverse ASTM 3039 specimen

$-V_{f}^{a} * N_{y}^{a}=V_{f}^{b} * N_{y}^{b}$

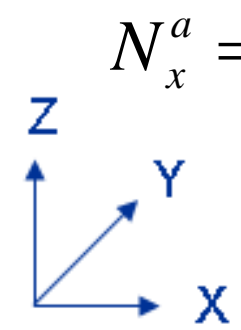

$N_{x}^{a}=N_{x}^{b}=N_{x}^{c}=N_{x}^{d}$

One Unit Cell

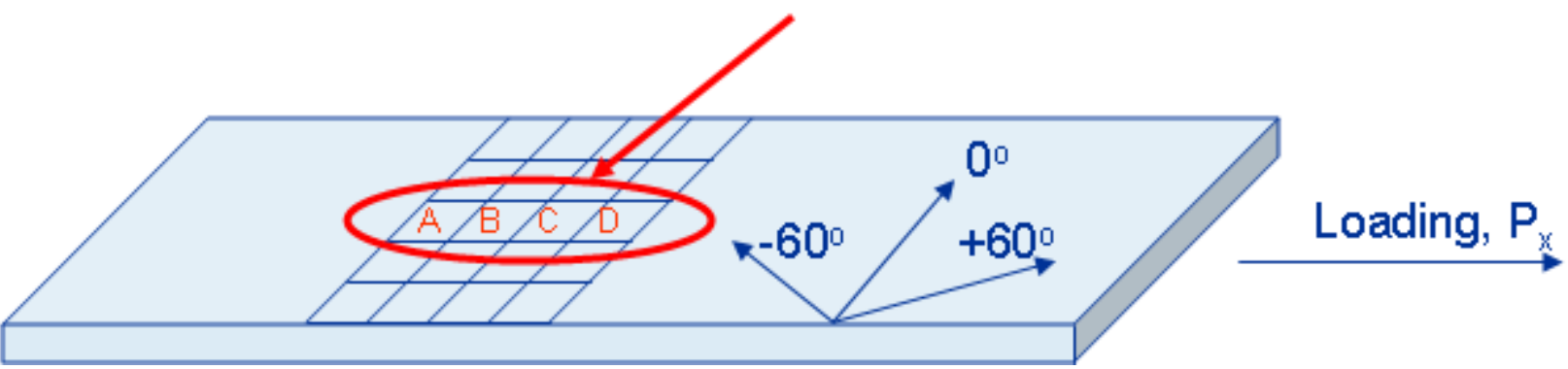

- Axial ASTM 3039 specimen

- $N_{y}^{a}=N_{y}^{b}=N_{y}^{c}=N_{y}^{d}=0$

$N_{y}^{a}=0=A 12^{a} * \varepsilon_{x}^{a}+A 22^{a} * \varepsilon_{y}^{a}$
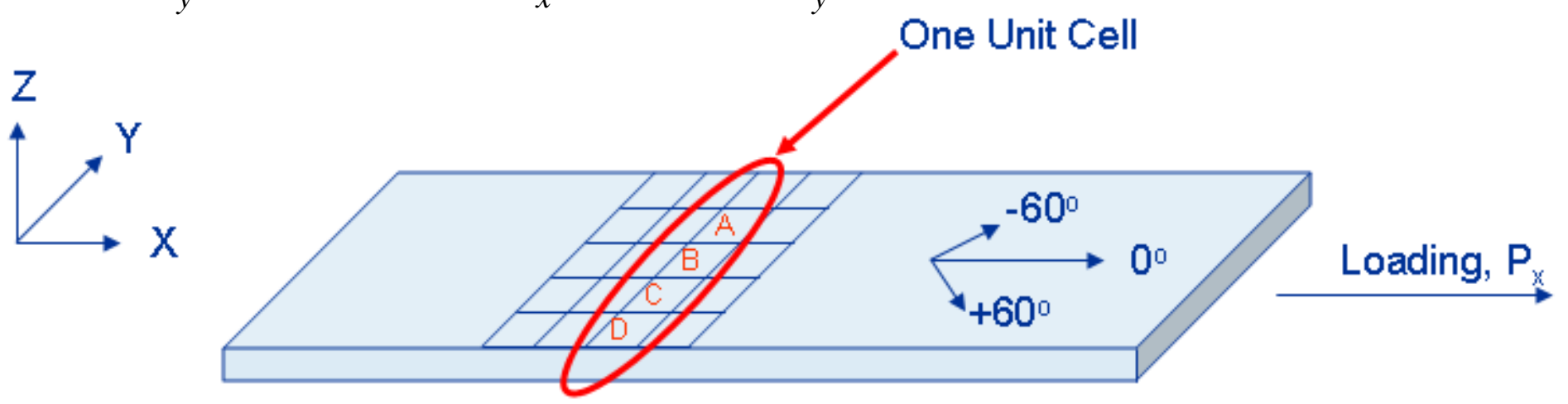


\section{Developed Equations (using Subcell Strains)}

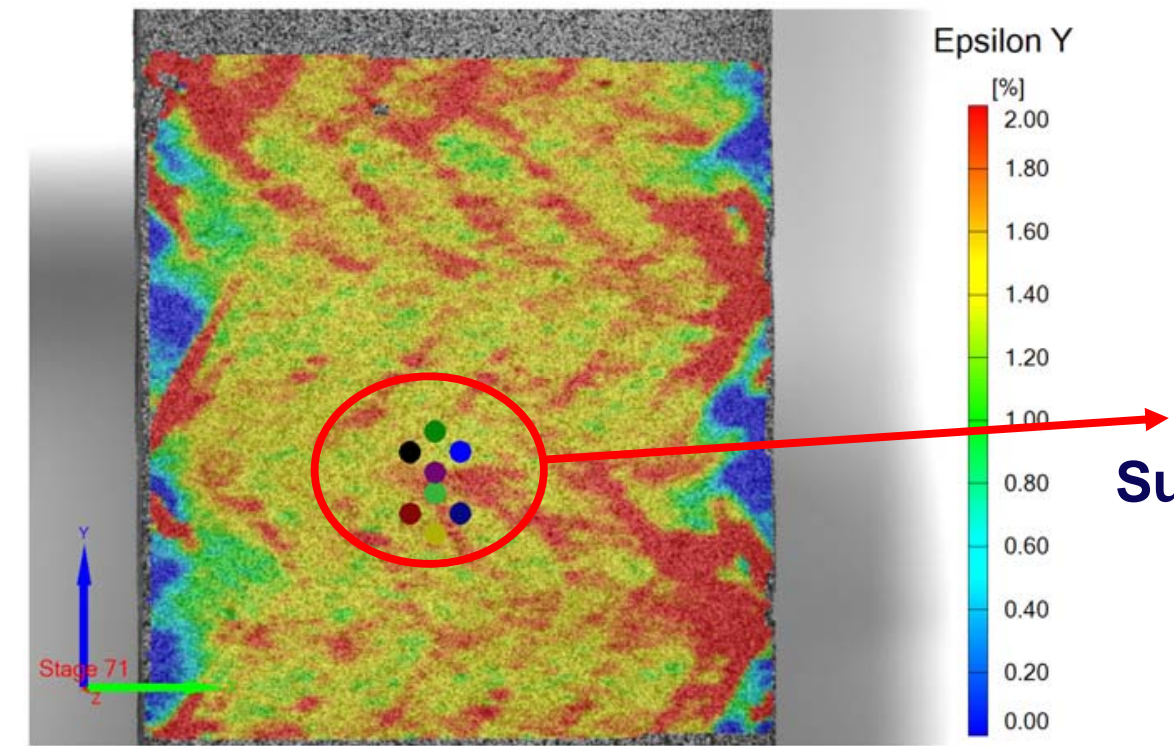

Transverse Tensile Test Global Axial Strain

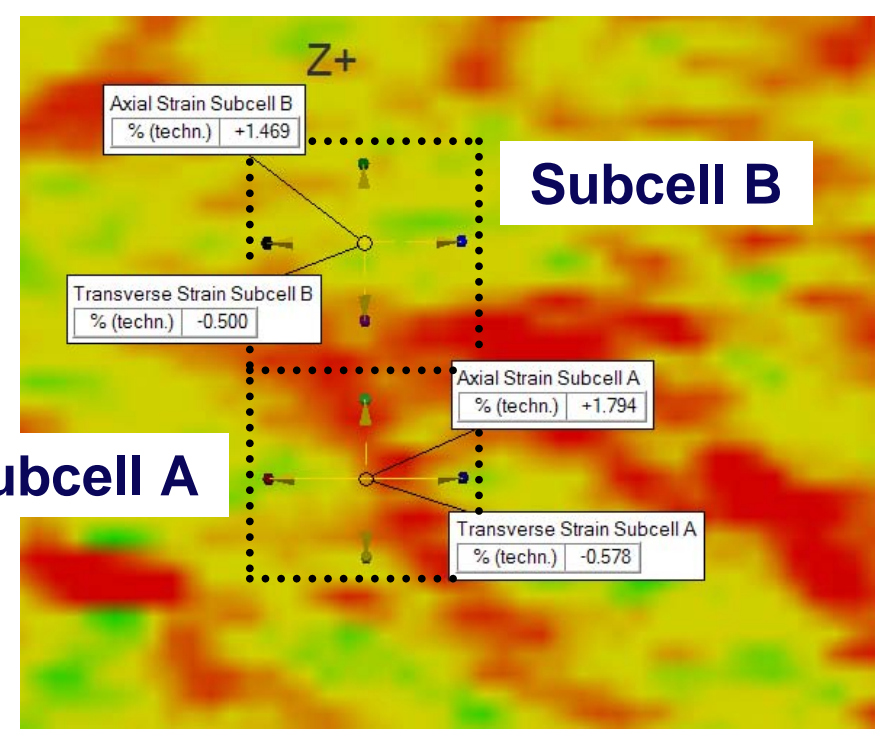

Local Subcell Strains

- Strains are found using the optical measurement system

- In the end, there are 6 variables (Q11,Q12,Q22,Q66, $\mathrm{Ny}^{\mathrm{a}}, \mathrm{Ny}^{\mathrm{b}}$ ) and 6 equations

- 2 from TT Subcells A and C (CLPT)

- 2 from TT Subcells B and D (CLPT)

- 1 from volume fraction averages (Micromechanics)

- 1 from AT Subcells A and C (CLPT)

- Solve simultaneously 


\section{Axial Tensile (AT) Strength}

- Assume that in AT tests, the AT fiber carries most of the load

- E11T comes from ultimate strain at AT failure

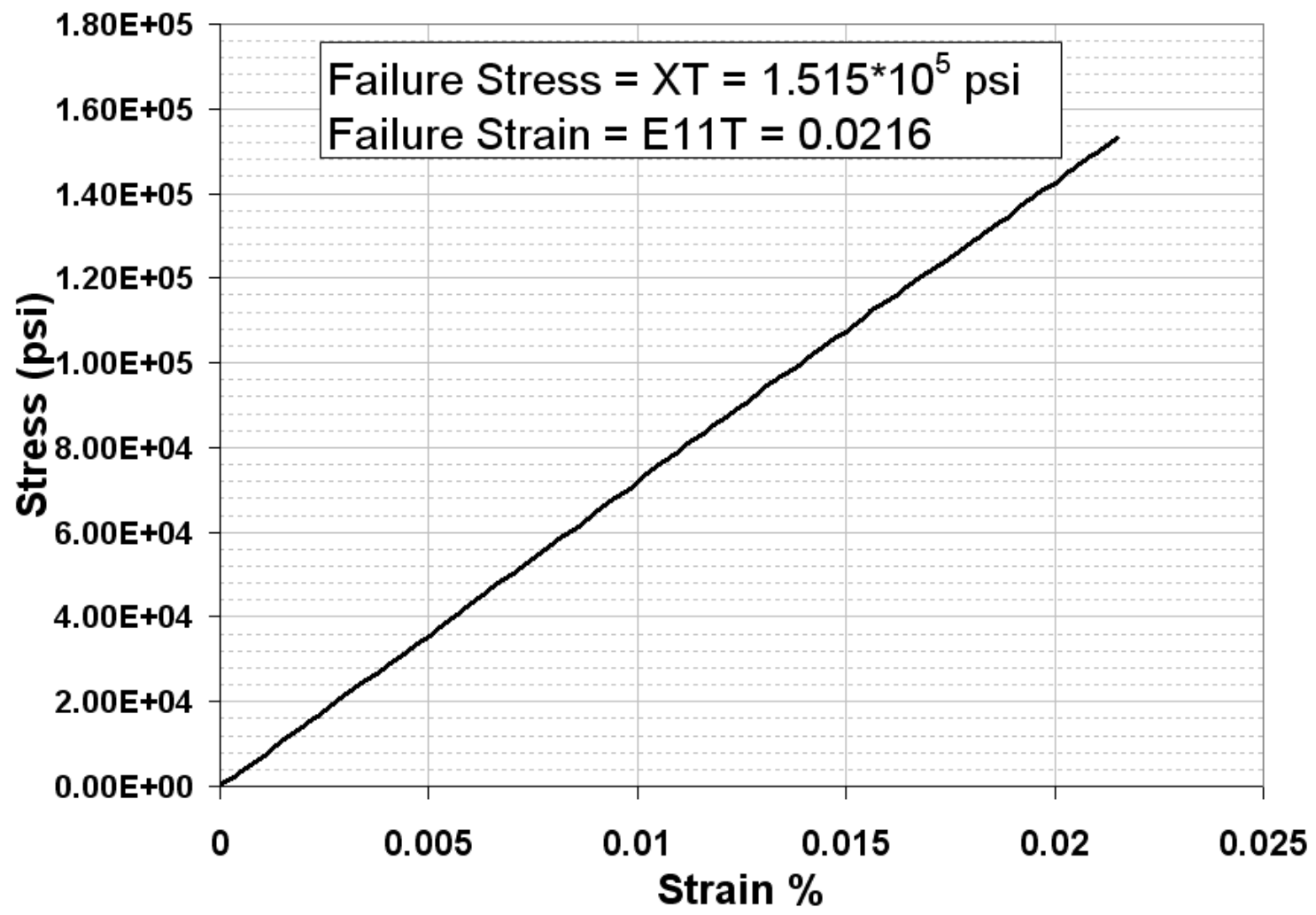




\section{Transverse Tensile (TT) Strength}

- Look at fiber splitting on TT specimen

- Load at first split will be YT

- SLIMT will be set to 1

- E22T will be set to failure strain of test

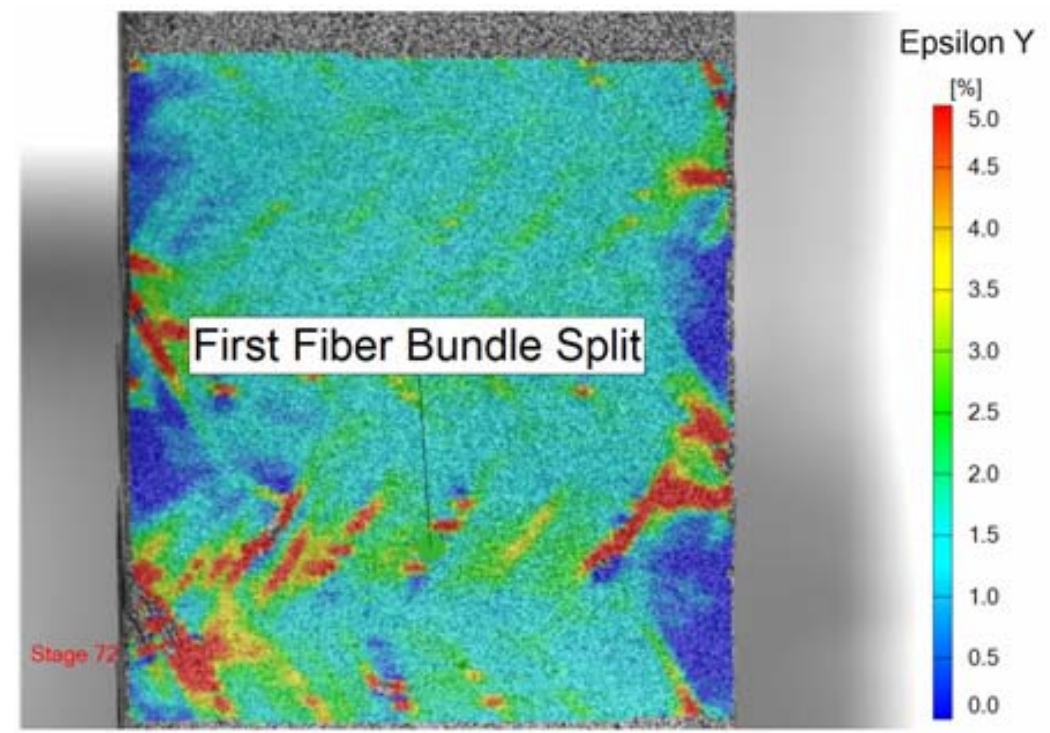

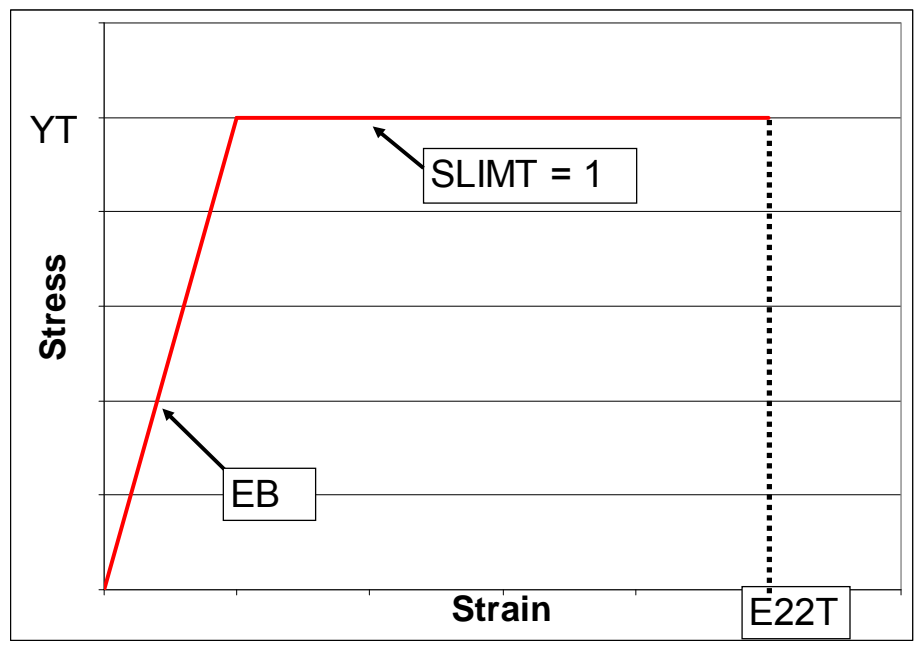

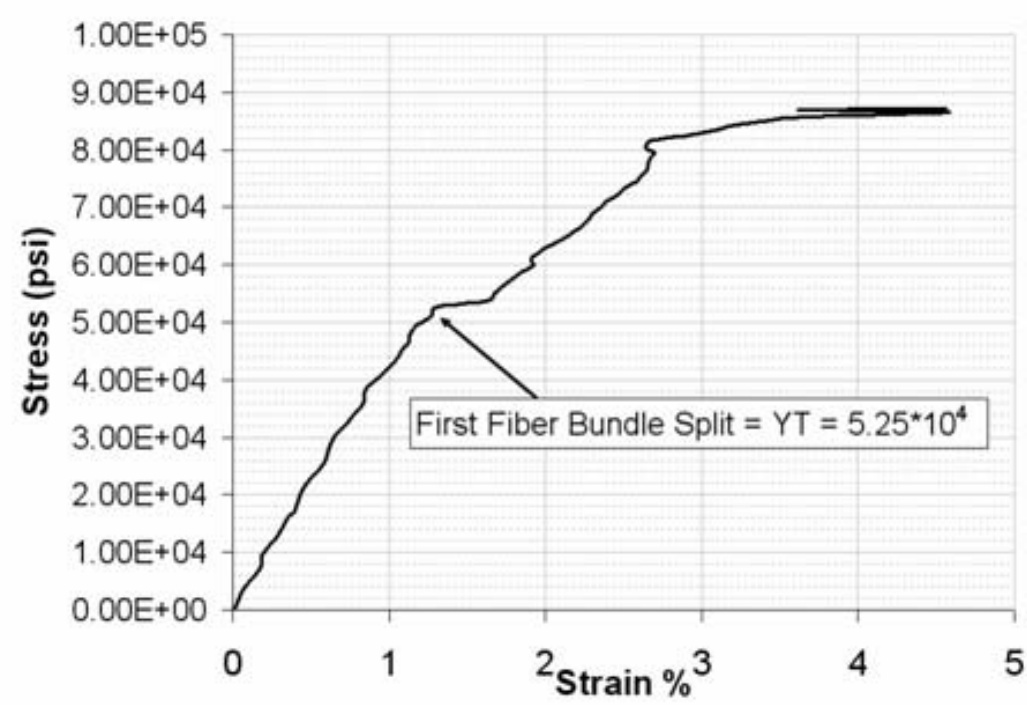




\section{Compressive Strain/Strength}

- Material behaves as a homogenous

- Use strength at failure for both Axial and Transverse tests

- Use strain at strength for both Axial and Transverse tests
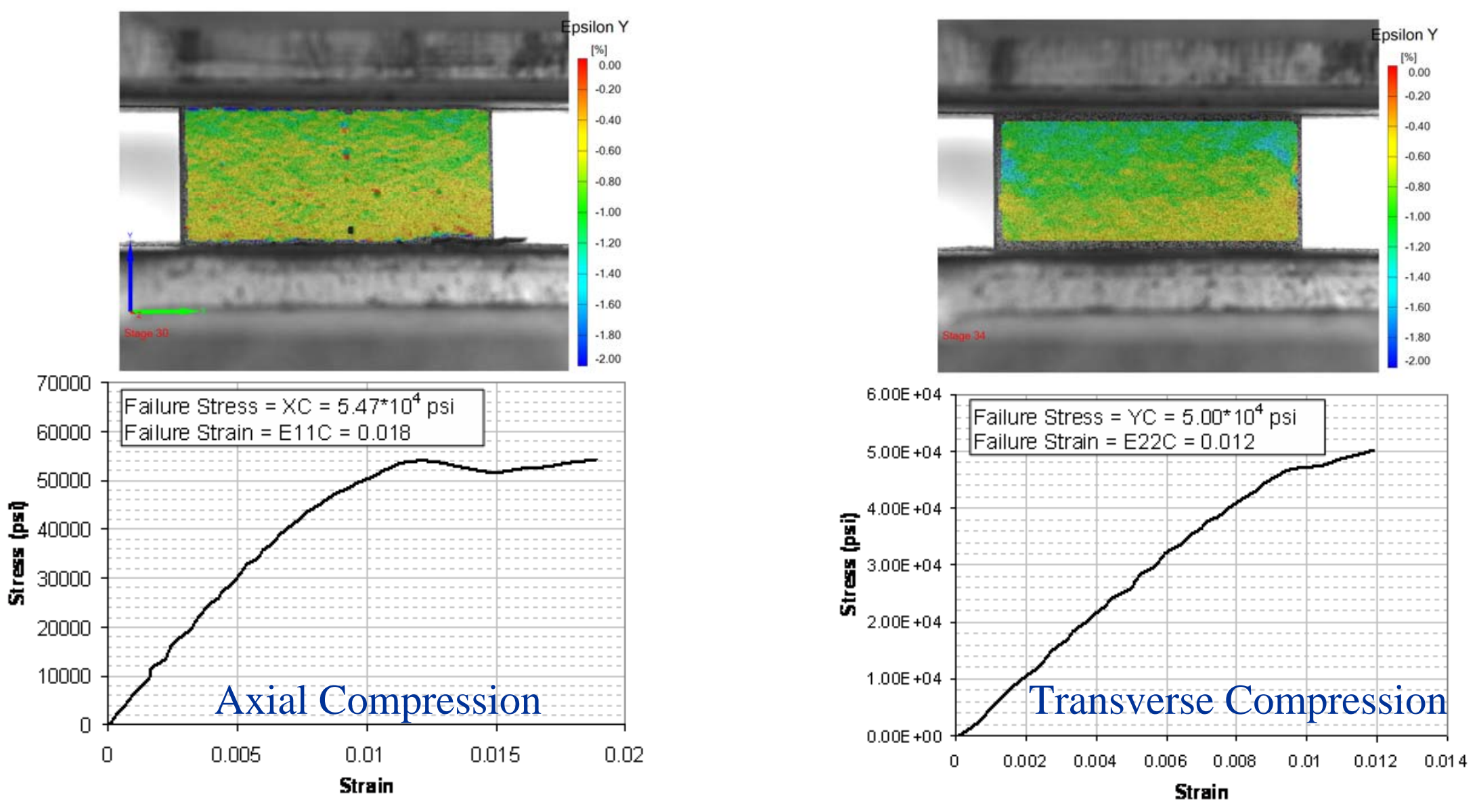


\section{Shear Strength}

- Using Modified Shear Specimen Design based on Kohlman

- ASTM 5379

- $P_{x y}, N_{x y}$ is for each of the integration layers

- Can be directly implemented for shear strength

- LS-DYNA needs $\varepsilon_{x y}$ for GMS
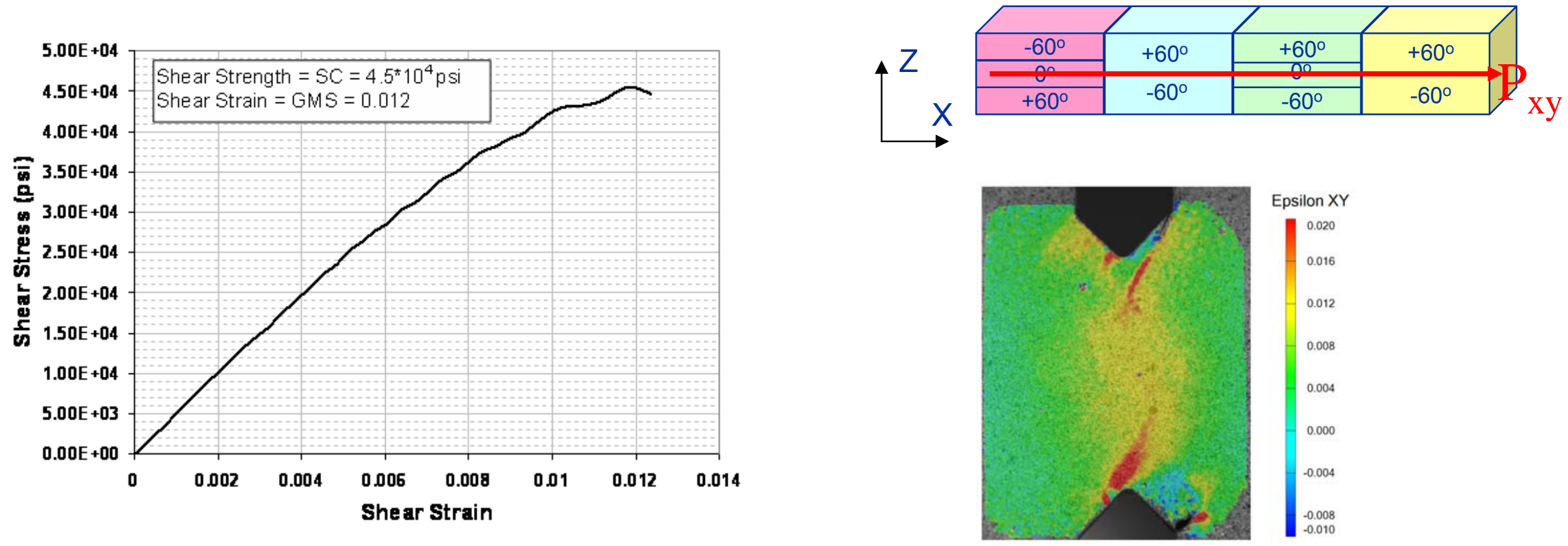


\section{Finite Element Models}
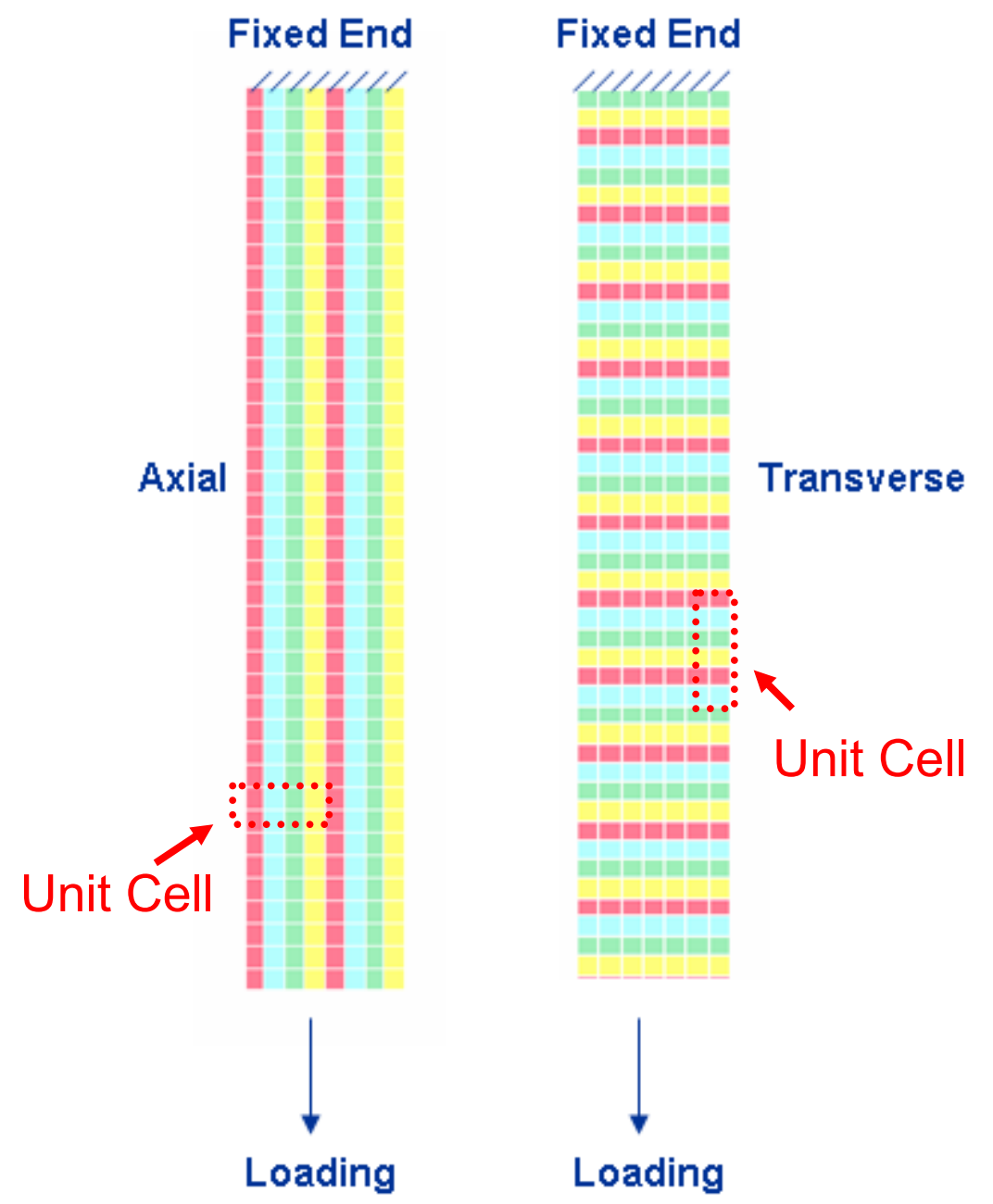

- Both Axial and Transverse Specimens were developed using ASTM D3039 specimen geometries

- Fixed end boundary conditions were used to simulate the fixed grip

- Loading was applied at the opposite end using enforced displacement 


\section{T700 / PR520}
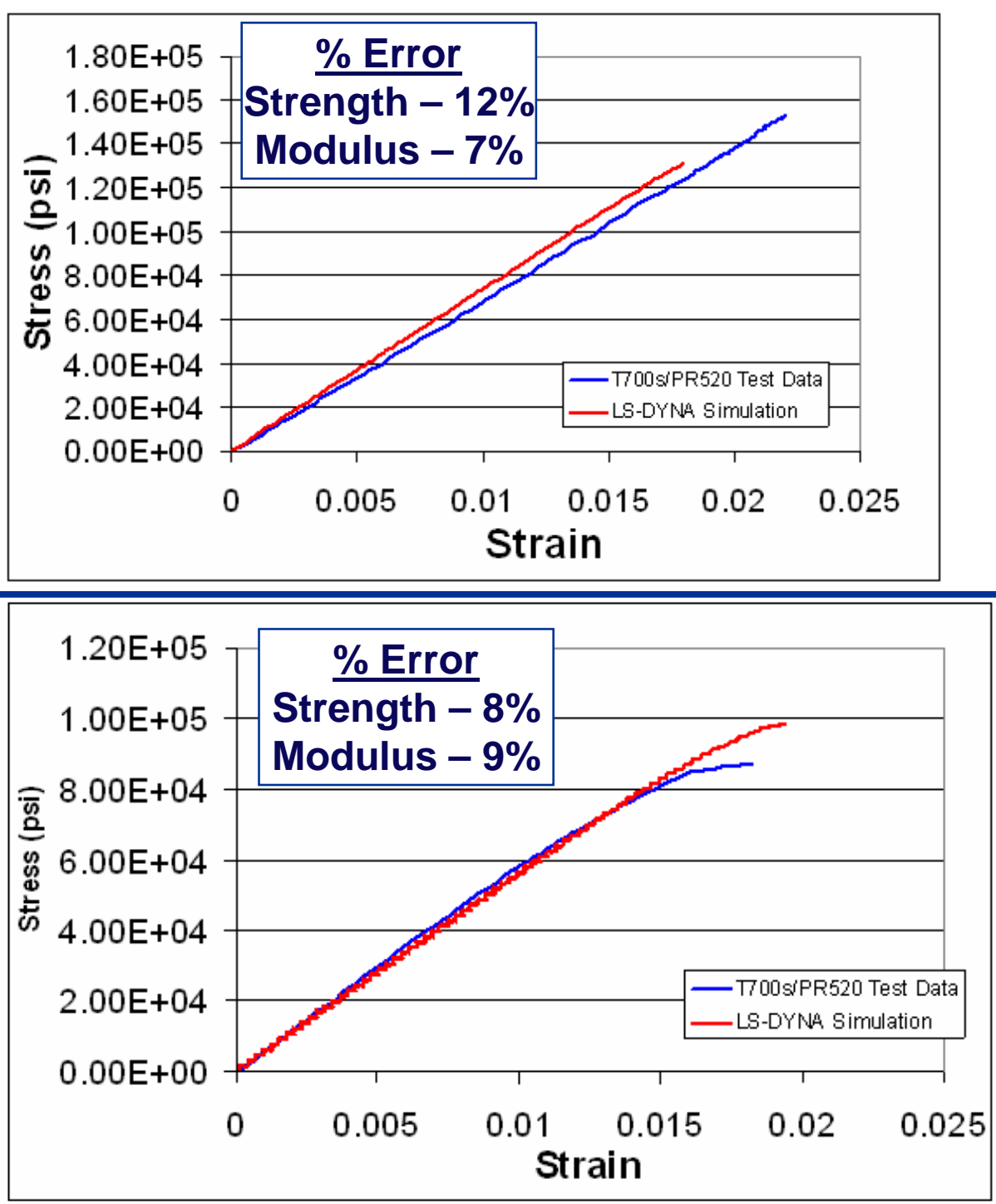

T700 / 3502
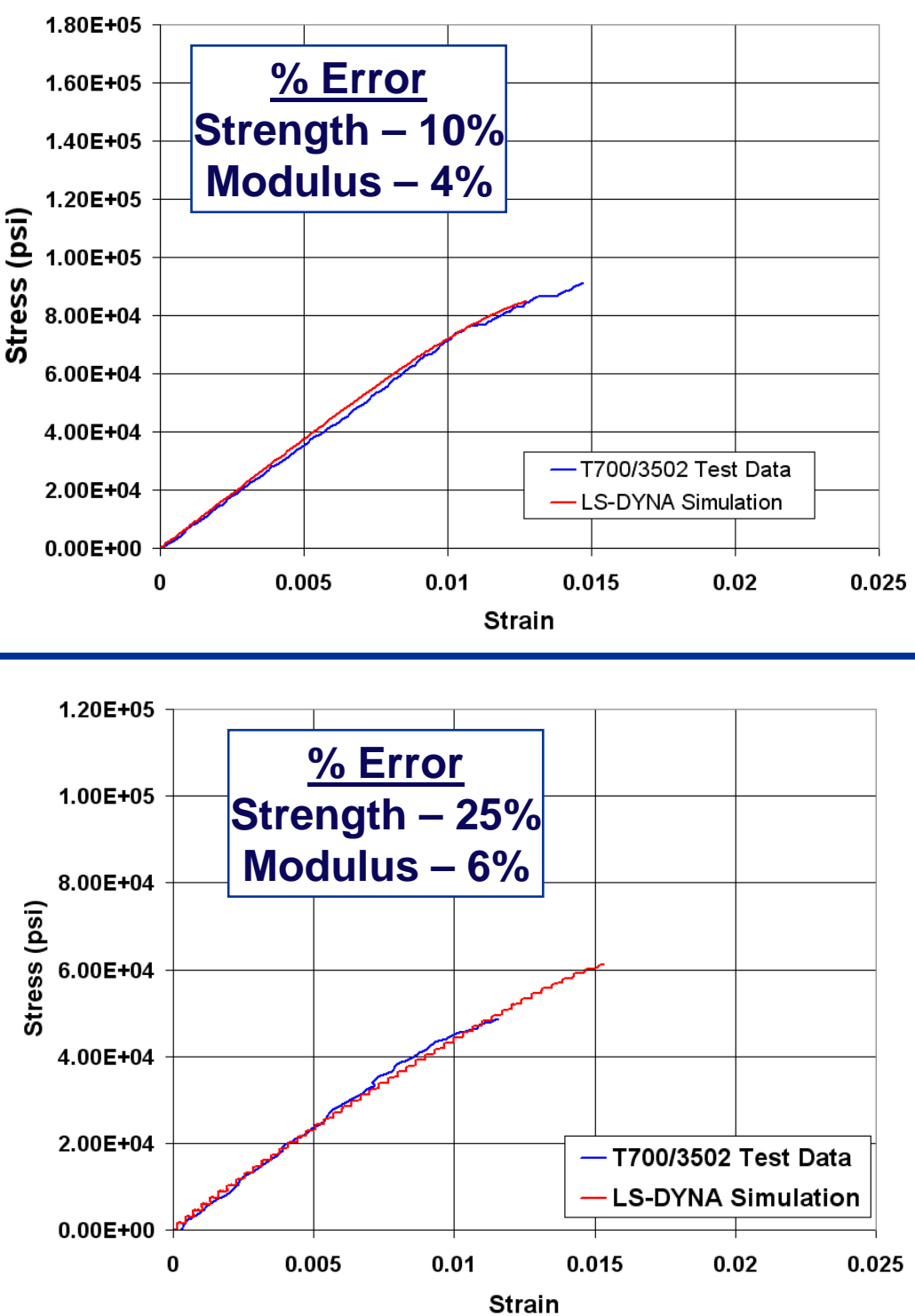


\section{Determination of T700 fiber / PR520 Resin Impact Characteristics}

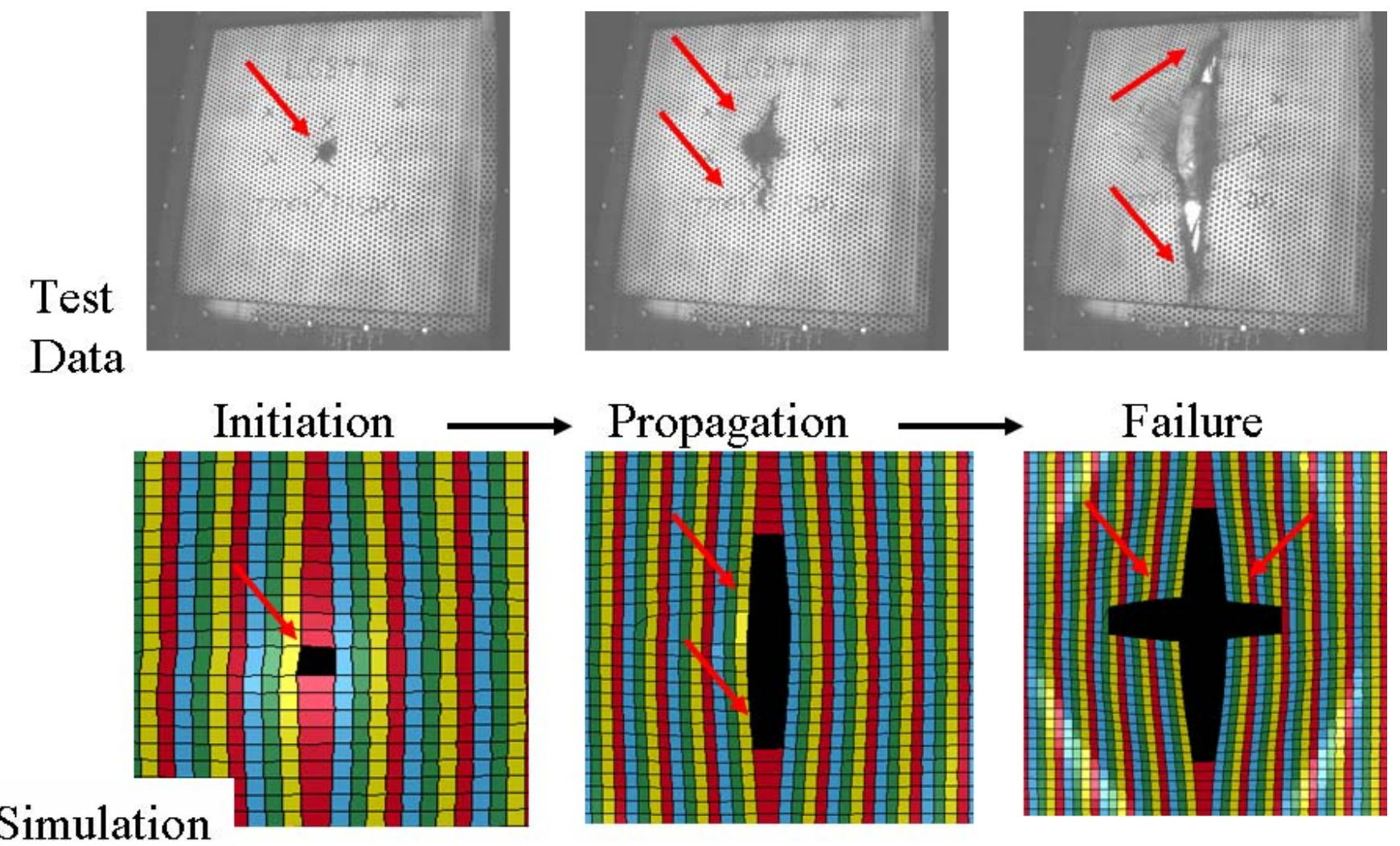

- Simulations were completed Spring 2008

- Showed penetration threshold at $630 \mathrm{ft} / \mathrm{sec}$

- Used as a starting point for impact tests

- Impact tests conducted Summer 2008

- Penetration threshold was between 609 and $637 \mathrm{ft} \mathrm{/} \mathrm{sec}$ 


\section{Limitations: Delaminations for T700 fiber / 3502 resin}

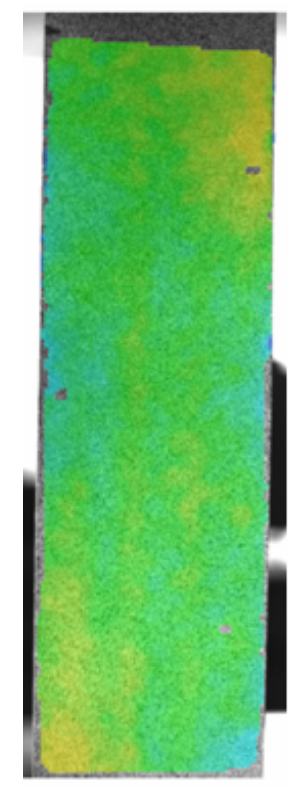

$50 \%$ $292 \mathrm{MPa}$

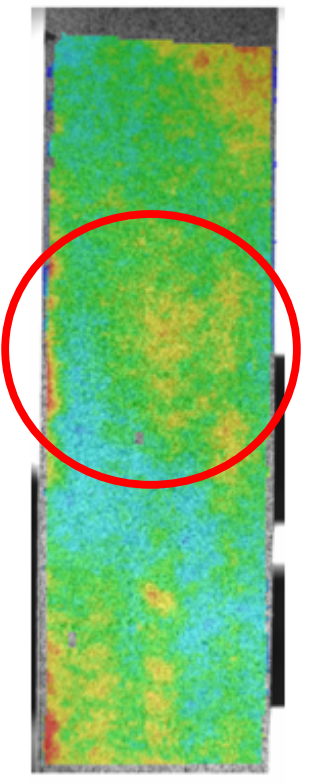

$75 \%$ $439 \mathrm{MPa}$

Out of Plane Deflection

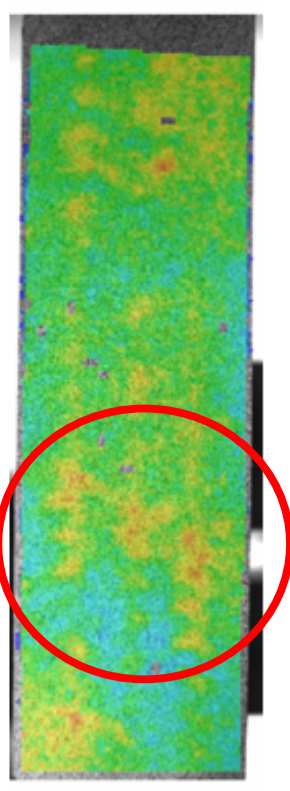

$90 \%$ $535 \mathrm{MPa}$

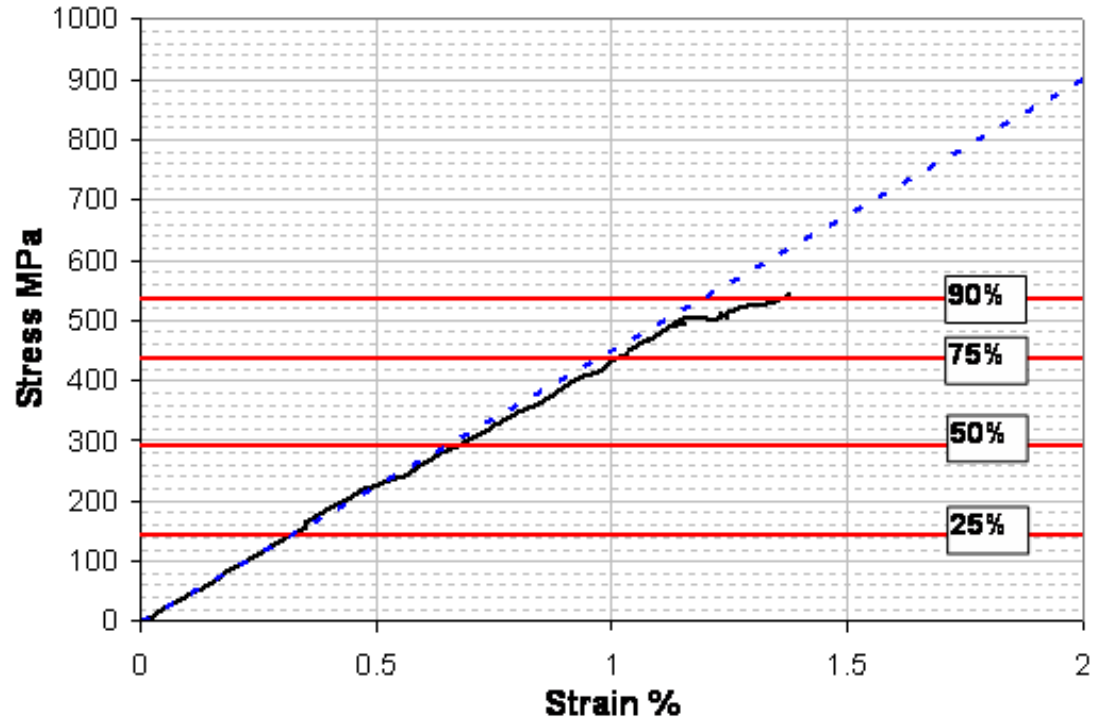

- OOP displacements verified by NDE

- Global Material response curves become non-linear after delaminations occurred

- Due to the nature of integration point formulation, cannot simulate ultimate failure values between layers 


\section{Limitations: FEM cannot simulate fiber bundle splitting}

- Axial Tension (axial strain)

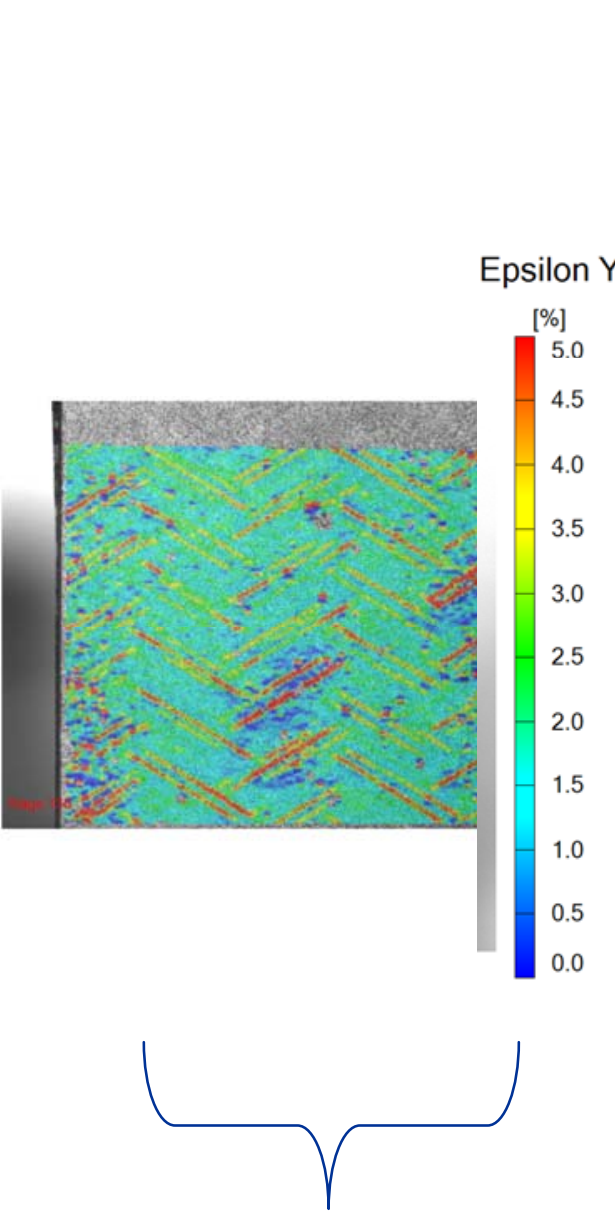

Optical Measurements

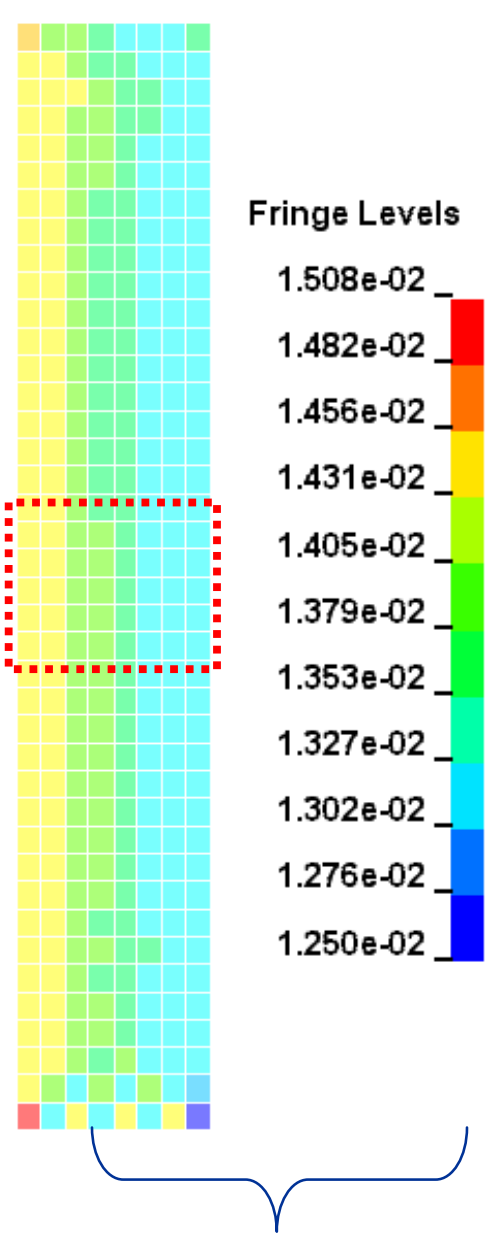

LS-DYNA
- Transverse Tension (axial strain)

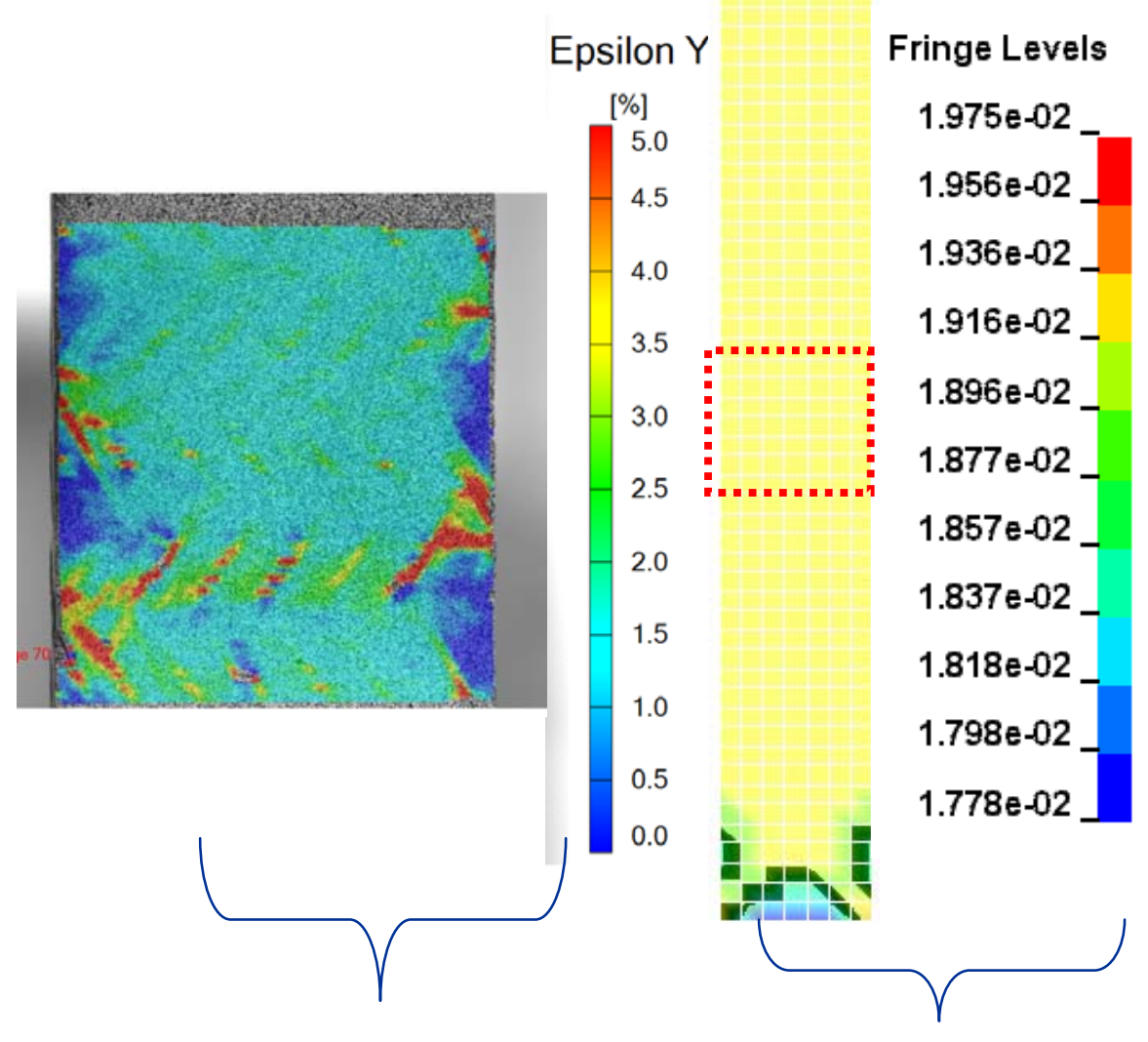

Optical Measurements 


\section{Conclusion}

- Standardized test methods in conjunction with an optical measurement system have been used to collect material property data for triaxial braided composite materials

- Global material response curves

- Local transverse fiber bundle splitting

- Local subsurface delaminations

- A hybrid micro-macromechanical computer model has been developed

- Incorporates braid architecture

- Incorporates tested material property data

- Comparisons between test and simulation show good agreement

- $10 \%$ in static simulations

- Penetration threshold in impact simulations

- Factors seen in the test data cannot be simulated as of now

- Ongoing work 


\section{Backup}




\section{Equation Development - Transverse Tensile Testing $_{\text {One Unit Cell }}$}
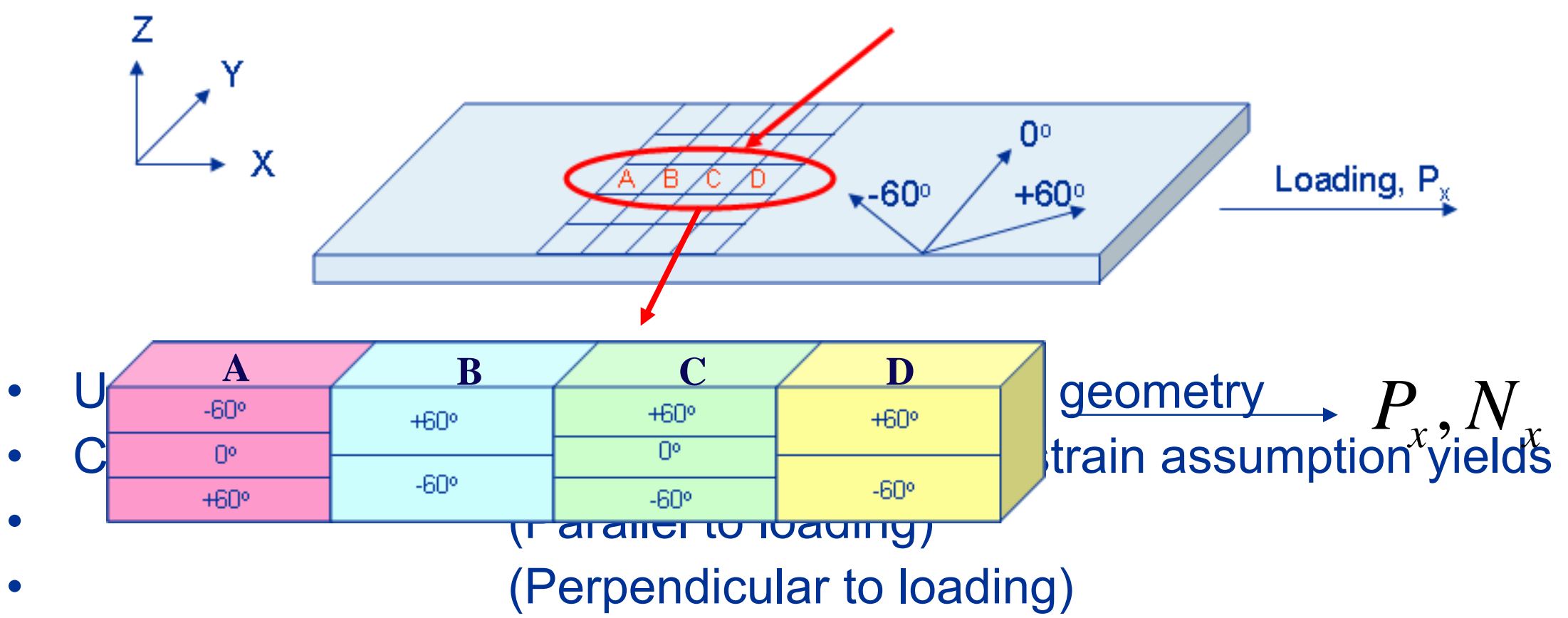

$$
\begin{gathered}
N_{x}^{a}=N_{x}^{b}=N_{x}^{c}=N_{x}^{d} \\
V_{f}^{a} * N_{y}^{a}=V_{f}^{b} * N_{y}^{b}
\end{gathered}
$$




\section{Rewriting the equations for each Subcell (Transverse Tensile Testing)}

- Subcell A

- $N_{x}$ is applied load and all strains are found from optical measurement system

$$
\begin{aligned}
& N_{x}^{a}=A 11^{a} * \varepsilon_{x}^{a}+A 12^{a} * \varepsilon_{y}^{a} \\
& N_{y}^{a}=A 12^{a} * \varepsilon_{x}^{a}+A 22^{a} * \varepsilon_{y}^{a}
\end{aligned}
$$

- Subcell B

- $\mathrm{N}_{\mathrm{x}}$ is applied load and all strains are found from optical measurement system

$$
\begin{aligned}
& N_{x}^{b}=A 11^{b} * \varepsilon_{x}^{b}+A 12^{b} * \varepsilon_{y}^{b} \\
& N_{y}^{b}=A 12^{b} * \varepsilon_{x}^{b}+A 22^{b} * \varepsilon_{y}^{b}
\end{aligned}
$$

- Four Equations 


\section{Equation Development - Axial Tensile Testing}

One Unit Cell

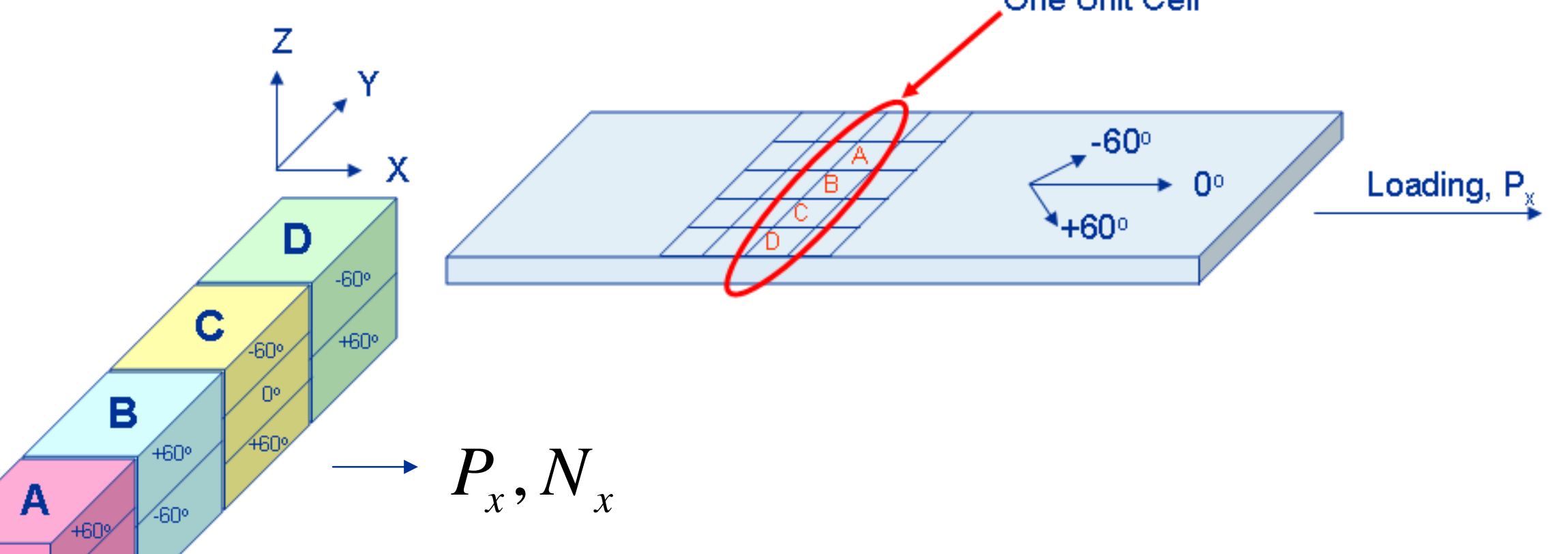

- Classical composite laminate micromechanics assumptions yield

$$
\begin{aligned}
& N_{y}^{a}=N_{y}^{b}=N_{y}^{c}=N_{y}^{d}=0 \\
& N_{y}^{a}=0=A 12^{a} * \varepsilon_{x}^{a}+A 22^{a} * \varepsilon_{y}^{a}
\end{aligned}
$$




\section{Advanced Data Analysis}

Toughened Fiber Bundle Splitting - Transverse Testing

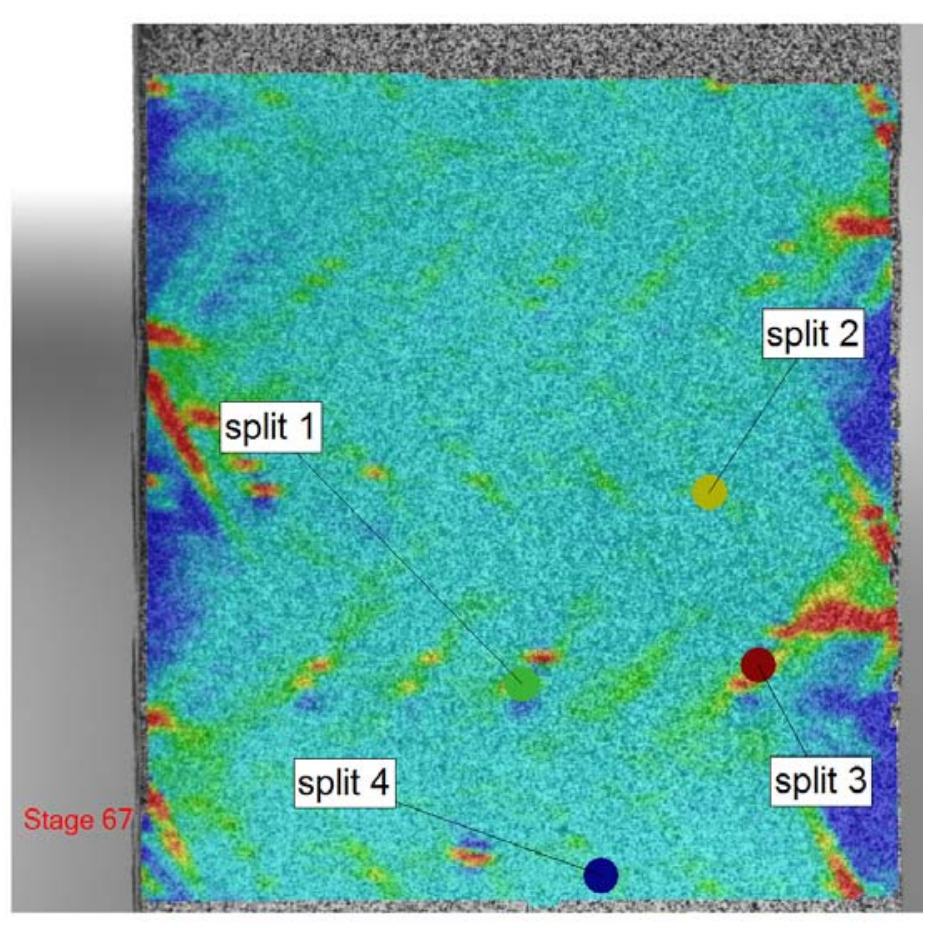

- Identify local failure strain

- Local failure initiation correlations to global nonlinearities
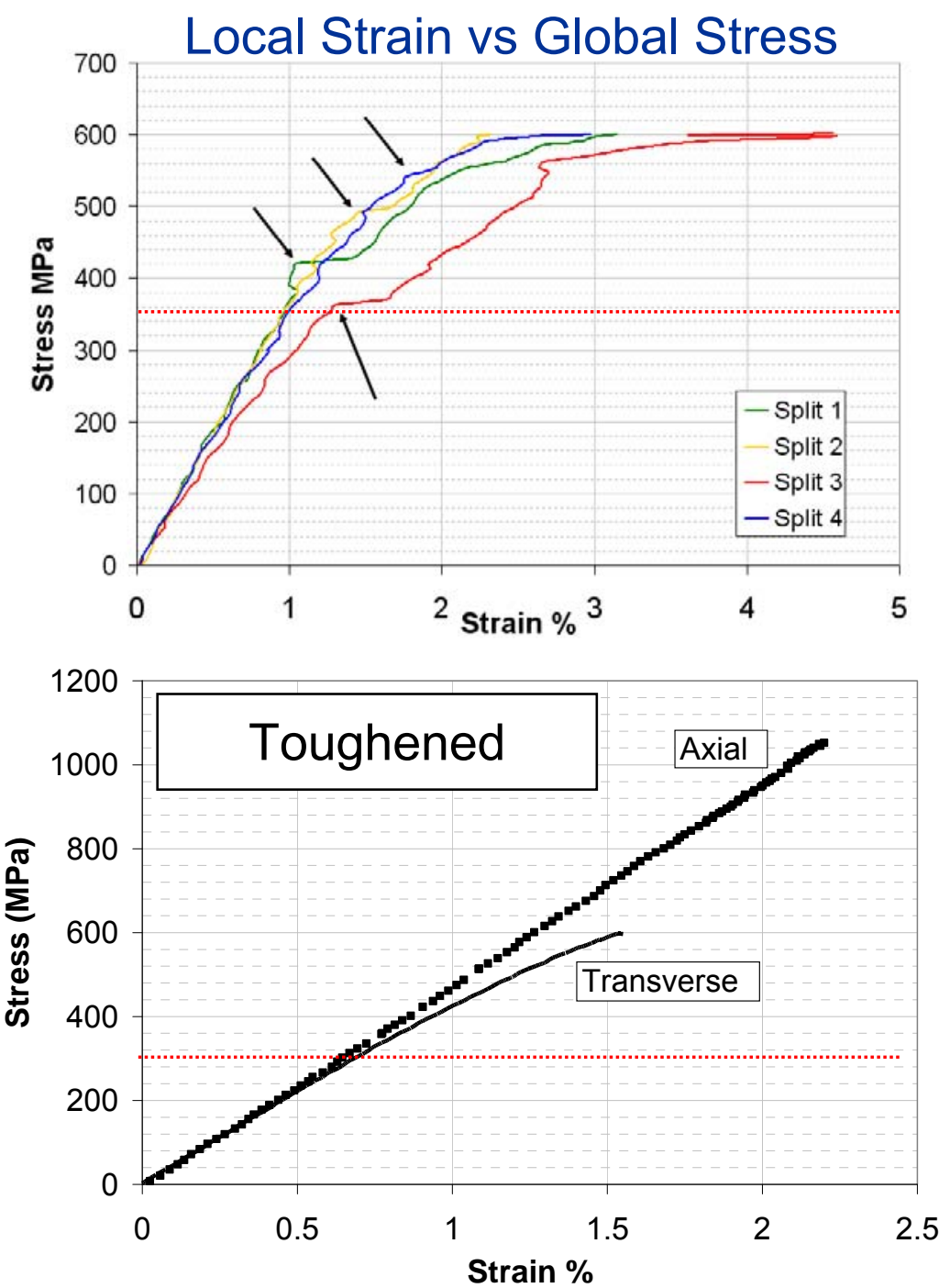


\section{T700 Fiber / PR520 Resin Static Results}

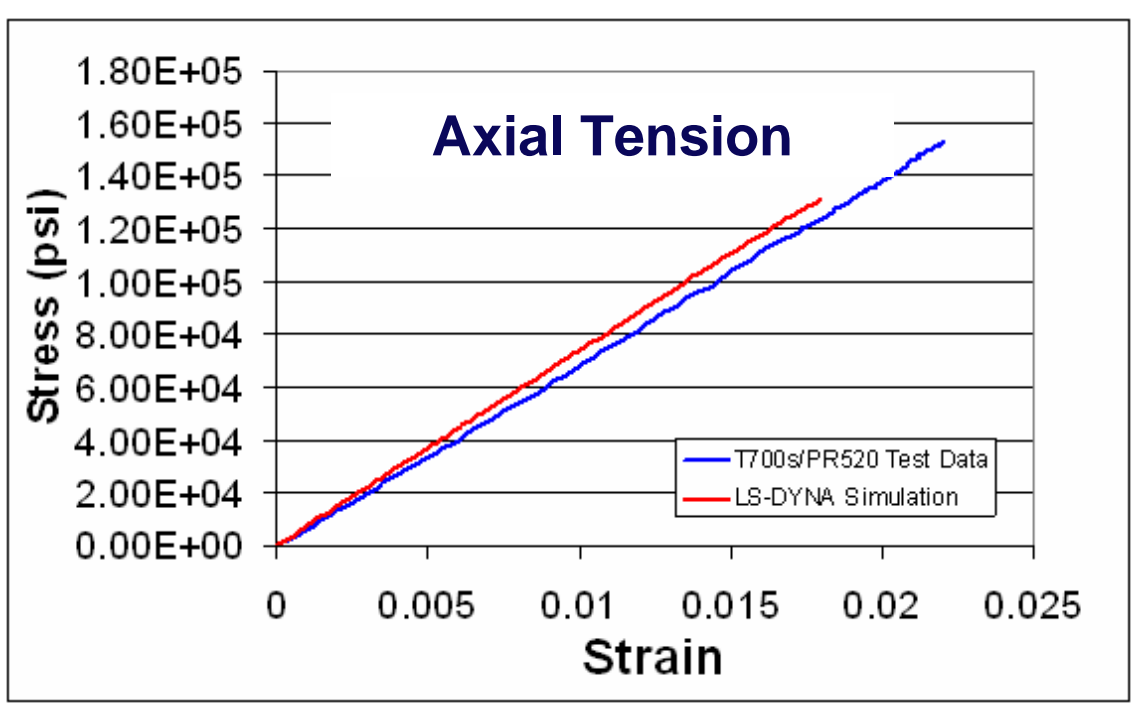

\begin{tabular}{|l|l|l|}
\hline & $\begin{array}{l}\text { Axial Tension } \\
\text { Modulus } \\
\text { (psi) }\end{array}$ & $\begin{array}{l}\text { Axial Tension } \\
\text { Strength } \\
\text { (psi) }\end{array}$ \\
\hline Test & $6.8 \mathrm{E} 6 \pm 1.6 \mathrm{E} 5$ & $1.52 \mathrm{E} 5 \pm 4.9 \mathrm{E} 3$ \\
\hline LS-DYNA & $7.4 \mathrm{E} 6$ & $1.31 \mathrm{E} 5$ \\
\hline$\%$ Error & $7 \%$ & $12 \%$ \\
\hline
\end{tabular}

\begin{tabular}{|l|l|l|}
\hline & $\begin{array}{l}\text { Transverse } \\
\text { Tension Modulus } \\
(\boldsymbol{p s i})\end{array}$ & $\begin{array}{l}\text { Transverse } \\
\text { Tension } \\
\text { Strength } \\
\text { (psi) }\end{array}$ \\
\hline Test & $6.2 \mathrm{E} 6 \pm 2.3 \mathrm{E} 5$ & $8.69 \mathrm{E} 4 \pm 4.3 \mathrm{E} 2$ \\
\hline LS-DYNA & $5.6 \mathrm{E} 6$ & $9.38 \mathrm{E} 4$ \\
\hline$\%$ Error & $9 \%$ & $8 \%$ \\
\hline
\end{tabular}

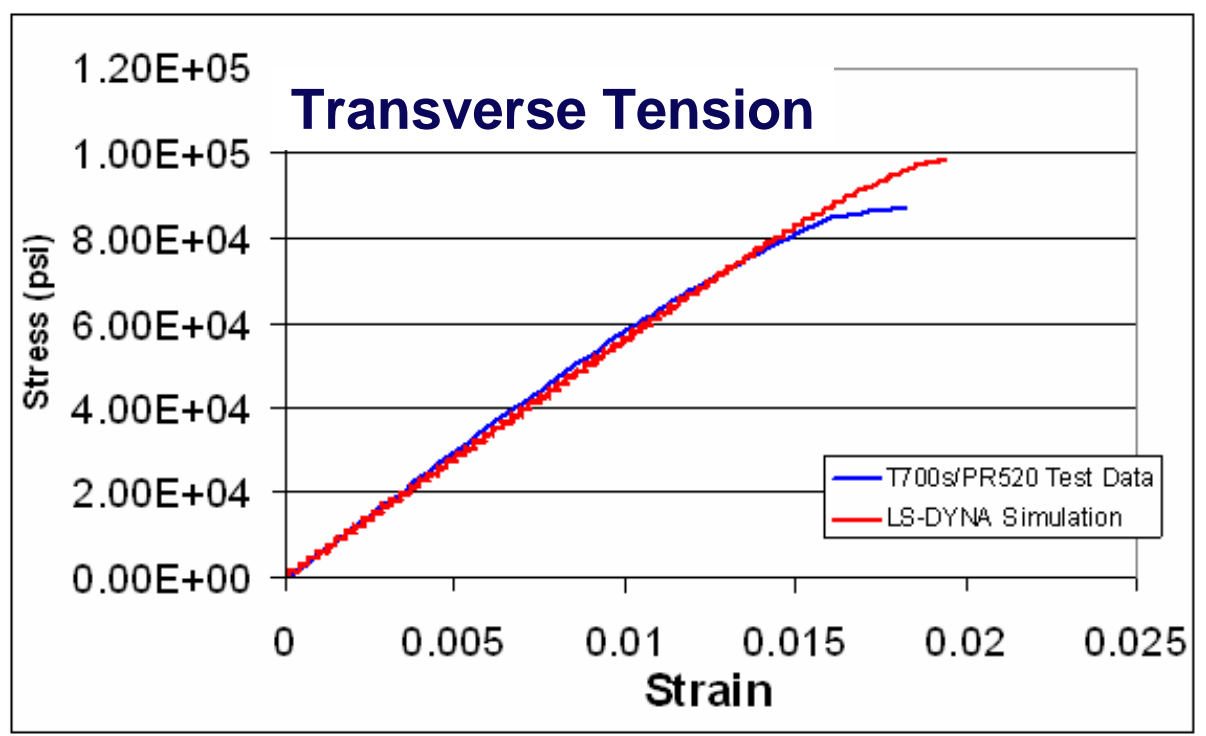

